\title{
Isolation and characterization of Newcastle disease virus from vaccinated commercial layer chicken
}

\author{
P. Balachandran ${ }^{1}$, P. Srinivasan ${ }^{2}$, S. Sivaseelan ${ }^{1}$, G. A. Balasubramaniam ${ }^{1}$ and T. R. Gopala Krishna Murthy ${ }^{2}$
}

\begin{abstract}
1. Department of Veterinary Pathology, Veterinary College and Research Institute, Namakkal, Tamil Nadu, India; 2. Poultry Disease Diagnosis and Surveillance Laboratory, Veterinary College and Research Institute, Namakkal, Tamil Nadu, India. Corresponding author: P. Srinivasan, email: srinipat2004@yahoo.com PB; balaavg@yahoo.co.in, SS; pathologysiva@yahoo.co.in, GAB; gabalasubramaniam@gmail.com,TRG; gkmurthy_in@yahoo.com Received: 11-04-2014, Revised: 31-05-2014, Accepted: 03-06-2014, Published online: 04-07-2014
\end{abstract}

doi: 10.14202/vetworld.2014.457-462 How to cite this article: Balachandran P, Srinivasan P, Sivaseelan S, Balasubramaniam GA and Gopala Krishna Murthy TR (2014) Isolation and characterization of Newcastle disease virus from vaccinated commercial layer chicken, Veterinary World 7(7): 457-462.

\begin{abstract}
Aim: Newcastle disease (ND) is an infectious, highly contagious and destructive viral disease of poultry and controlled by vaccination. In spite of vaccination, incidence of ND was reported in commercial layers with gastrointestinal lesions. This study was undertaken to assess the prevalence and pathotypes of Newcastle disease virus (NDV) involved in gastrointestinal tract abnormalities of vaccinated commercial layer chicken of Namakkal region for a period of three years from 2008 and 2011.

Materials and Methods: Pooled tissue (trachea, lung, spleen, proventriculus, intestine and caecal tonsils) samples collected from dead birds on postmortem examination from 100 layer flocks above 20 weeks of age with gastrointestinal lesions were subjected to isolation of NDV in embryonated specific pathogen free (SPF) chicken eggs. Mean death time (MDT) and intracerebral pathogenicity index of the isolates were characterized. Flock details were collected from NDV positive flocks to assess the prevalence and impact of NDV on vaccinated commercial layer chicken.

Results: Among the 100 flocks examined Newcastle disease virus was detected in 14 flocks as a single infection and 10 flocks as combined infections with worm infestation, necrotic enteritis and coccidiosis. Chicken embryo mean death time (MDT) and intracerebral pathogenicity index (ICPI) values ranged from 50.4 to $96.0 \mathrm{hrs}$ and from 0.650 to 1.675 respectively. Affected birds showed anorexia, diarrohea and drop in egg production. Macropathologically, matting of vent feathers, petechial haemorrhage on the tip of proventricular papilla, caecal tonsils and degeneration of ovarian follicles were noticed. The incidence of ND was most commonly noticed in 20-50 wk of age and between the months of September to November. Morbidity rate varied from $5 \%$ to $10 \%$ in the NDV alone affected flocks and 5 to $15 \%$ in NDV with other concurrent infections. Egg production drop from the expected level ranged between 3 to $7 \%$ in ND and 5 to $10 \%$ in concurrent infections. Average mortality in NDV and concurrently affected (NDV and Coccidiosis) flocks were $2.89 \%$ and $3.50 \%$ respectively.

Conclusion: The present study revealed $24 \%$ of gastrointestinal tract abnormalities in commercial layer chicken were caused by various pathotypes of Newcastle disease virus. The virus caused the disease as single and concurrently with other diseases. Vaccination minimized the clinical manifestation and lesions even in velogenic virus affected flocks.
\end{abstract}

Keywords: commercial layer chicken, Newcastle disease virus, pathology, prevalence.

\section{Introduction}

Newcastle disease (ND) is a highly contagious and wide spread viral disease of the avian species causing severe economic losses in domestic poultry, especially chickens [1]. This disease is still one of the most important diseases in poultry production worldwide and remains a major constraint against the development of both industrial and village poultry production in Asia in spite of control measures including vaccination which has been applied since 1950s. Its global impact is enormous and remains a major barrier to international trade in poultry and poultry products and a greater drain on the world economy. The causative agent of the disease is Newcastle disease virus (NDV) also designated as Avian paramyxovirus serotype 1, which is a negativesense single-stranded RNA virus and belongs to the

Copyright: The authors. This article is an open access article licensed under the terms of the Creative Commons Attribution License (http://creativecommons.org/licenses/by/2.0) which permits unrestricted use, distribution and reproduction in any medium, provided the work is properly cited. genus Avulavirus within the family Paramyxoviridae [2].

The disease can vary from clinically in apparent to highly virulent forms, depending on the virus strain and the host species. NDV detection and pathotyping of avian isolates are extremely important because the appearance of virulent virus has significant economic consequences related to vaccination and eradication, impairing the ability of a given geographic region to export poultry products [3]. The widespread presence of lentogenic strains in feral birds and the use of such viruses as live vaccines mean that isolation of NDV is not enough to confirm a disease diagnosis and compliance with statutory requirements that may be in place [4]. Viral characterization using the pathogenicity test or nucleotide sequencing is also required, as the importance and impact of a given NDV isolate are directly related to its virulence. Hence analysis of a given field disease solely may be an unreliable measurement of pathogenicity due to the possibility of concurrent infections and bad technical management, laboratory assessments of the virus pathogenicity are necessary. 
For this purpose, currently three "in vivo" tests are available, which include determination of Intracerebral Pathogenicity Index (ICPI), Intravenous Pathogenicity Index (IVPI) and Mean Death Time (MDT) [2].

Namakkal is the most thickly populated poultry zone in India with a layer population of 45 million birds and occupying second place in egg production at national level [5]. In the recent past, due to strict vaccination policy and improved hygienic measures, outbreaks of NDV were mild without the typical clinical and pathological manifestations of ND such as acute diarrhea or dysponea and haemorrhgic enteritis or tracheitis in commercial layers, however drop in egg production with mild haemorrhages in the proventricular papillae and enteritis were commonly noticed. Characterization and pathotyping of NDV from such cases are extremely important because of the extreme variation in the virulence of different NDV isolates and the widespread use of live vaccines in this region [2].

However information on the pathotype of NDV in vaccinated commercial layers above 20 weeks of age with gastrointestinal lesions is meager. Hence, this study was undertaken to assess the prevalence and pathotype of NDV in gastrointestinal lesions of vaccinated commercial layer chickens.

\section{Materials and Methods}

Data collection: The prevalence of NDV in commercial layer chickens with gastrointestinal tract abnormalities was studied for a period of 3 years (October 2008 to September 2011). One hundred layer flocks with history and symptoms suggestive of gastrointestinal tract infection were inspected and the information regarding breed and strain of chicken, flock strength, age, method of rearing, vaccination schedule, production performance, symptoms manifested and mortality were collected. The seasons were classified as summer (March, April and May), south west monsoon (June, July and August), north east monsoon (September, October and November) and winter (December, January and February). The age was classified into six groups viz., 21-30 wk, 31- 40 wk, 41-50 wk, 51-60 wk, 61-70 wk and 71-80 wk [5].

Virus isolation: The dead birds were subjected to detailed postmortem examination as per approved procedure. Organs were examined systematically for gross pathological changes. During postmortem examination, concurrent infections such as worm infestation, necrotic enteritis and coccidiosis were diagnosed based on the gross lesions and laboratory tests. Pooled tissues (trachea, lung, spleen, proventriculus, intestine and ceacal tonsils) samples from each flock was finely minced and 20\% suspension was made in PBS containing Penicillin (2000 IU/ml) and Streptomycin $(2 \mathrm{mg} / \mathrm{ml})$. The samples were cleared by centrifugation at 2000 rpm for $15 \mathrm{~min}$. The supernatant was collected and subjected to haemagglutination (HA) test for detection of Newcastle disease virus (NDV) as per the method described by OIE [6]. A volume of $0.2 \mathrm{ml}$ of supernatant showing HA activity was injected into the allantoic cavity of five numbers of nine day old embryonated specific pathogen free (SPF) chicken eggs and incubated at $37^{\circ} \mathrm{C}$ for 7 days. Eggs containing dead embryos and all remaining eggs at the end of the incubation period were chilled to $4^{\circ} \mathrm{C}$ for overnight and the allontoic fluid was tested for presence of NDV by HA and haemagglutination inhibition (HI) tests. Those samples which were found negative for NDV at $3^{\text {rd }}$ passage were further passaged twice before being discarded as negative [6].

Biological characterization of NDV: Mean death time was performed in 10 day - old SPF embryonated eggs. Serial tenfold dilution of allantoic fluid $\left(10^{-1}\right.$ to $\left.10^{-9}\right)$ was prepared with sterile isotonic saline without any antibiotics. $0.1 \mathrm{ml}$ of allantoic fluid was inoculated into the allantoic cavity using a minimum 5 eggs per dilution and incubated at $37^{\circ} \mathrm{C}$. The eggs were examined twice daily for maximum period of 7 days by candling the eggs and embryo death was recorded. The highest dilution at which all embryos died was taken as mean lethal dose (MLD) and the MDT was calculated as the average time at which the eggs inoculated with MLD were died [7].

Intracerebral Pathogenicity Index (ICPI) was carried out in one day-old chicks from SPF parents. In the ICPI test, birds are inoculated intracerebrally and then examined every 24 hour for eight days. The birds are scored at each observation as 0 -if normal, 1 -if sick and 2-if dead. The ICPI is the mean score per bird per observation over the eight day period [6].

\section{Results}

Among the 100 flocks examined for gastrointestinal lesions, 24 were found to be positive for NDV. The HA titre of the pooled organ samples varied between 32 and 128 and it was inhibited by NDV reference antiserum, which proved the presence of the ND virus. Inoculation of pooled organ samples into the $9^{\text {th }}$ day old embryonated chicken eggs revealed embryo mortality, varying degrees of haemorrhages throughout the body including haemorrhages at the occipital region 3-7 days post inoculation. The virus was further confirmed in the allontoic fluid by NDV specific antiserum in HI test. The isolation in $66.67 \%$ of samples were positive in the first passage, $20.83 \%$ in the $2^{\text {nd }}$ passage and $12.50 \%$ in the $3^{\text {rd }}$ passage. The MDT observed in SPF chicken eggs were above 90, 6090 and below $60 \mathrm{hr}$ in $8.33,75.0$ and $16.67 \%$ of NDV isolates respectively. The ICPI value of the NDV isolates in day old SPF chicks were 0.65 to $0.963,1.013$ to 1.438 and 1.575 to 1.675 in $41.67,37.5$ and $20.83 \%$ (Table-1).

In ND affected flocks, the birds showed loss of appetite, depression, drop in egg production, drooping of the wings and head, cyanosis of comb and wattles and whitish to greenish diarrhoea (Figure-1). Manifestation of clinical signs varied from mild to moderate and few to many in the affected flocks. Pathologically, matting of vent feathers by green or white tinged faecal 
Table-1: Clinical history and biological characteristics of NDV isolated from flocks with gastrointestinal tract lesions.

\begin{tabular}{|c|c|c|c|c|c|c|c|c|}
\hline Flock no. & Age (week) & Flock size & Morbidity (\%) & Egg production drop (\%) & Mortality (\%) & Diagnosis & MDT hrs & ICPI \\
\hline 1. & 53 & 15,000 & 7.0 & 5.0 & 2.44 & ND & 81.6 & 0.813 \\
\hline 2. & 25 & 20,000 & 7.0 & 5.0 & 1.45 & ND & 55.2 & 1.625 \\
\hline 3. & 64 & 8,000 & 8.0 & 6.0 & 2.46 & ND & 81.6 & 0.763 \\
\hline 4. & 64 & 25,000 & 9.0 & 7.0 & 2.55 & ND & 74.4 & 0.963 \\
\hline 5. & 52 & 52,500 & 8.0 & 6.0 & 4.18 & ND & 72.0 & 1.175 \\
\hline 6. & 30 & 20,000 & 5.0 & 6.0 & 1.53 & ND & 62.6 & 1.213 \\
\hline 7. & 61 & 44,000 & 7.0 & 7.0 & 3.13 & ND & 79.2 & 0.850 \\
\hline 8. & 35 & 6,000 & 6.0 & 5.0 & 2.82 & ND & 67.2 & 1.288 \\
\hline 9. & 55 & 40,000 & 10.0 & 7.0 & 4.83 & ND & 72.0 & 1.113 \\
\hline 10. & 47 & 50,000 & 8.0 & 5.0 & 2.93 & ND & 72.2 & 1.075 \\
\hline 11. & 22 & 20,000 & -- & 6.0 & 2.79 & ND & 57.6 & 1.675 \\
\hline 12. & 32 & 20,000 & 7.0 & 7.0 & 3.89 & ND & 69.6 & 1.400 \\
\hline 13. & 30 & 5,000 & 5.0 & 4.0 & 1.94 & ND & 69.6 & 1.363 \\
\hline 14. & 47 & 5,000 & 8.0 & 6.0 & 3.52 & ND & 74.4 & 1.013 \\
\hline 15. & 26 & 5,000 & -- & 5.0 & 1.88 & $N D+R W$ & 50.4 & 1.663 \\
\hline 16. & 21 & 6,000 & 5.0 & 7.0 & 2.33 & ND +RW & 76.8 & 0.925 \\
\hline 17. & 28 & 9,000 & -- & 7.0 & 1.94 & ND+TW & 52.2 & 1.625 \\
\hline 18. & 47 & 50,000 & 10.0 & 8.0 & 3.42 & ND+TW & 86.4 & 0.750 \\
\hline 19. & 24 & 30,000 & 5.0 & 7.0 & 1.69 & ND+TW & 60.0 & 1.575 \\
\hline 20. & 42 & 25,000 & 9.0 & 6.0 & 3.67 & ND+TW & 64.6 & 1.438 \\
\hline 21. & 55 & 10,000 & 12.0 & 9.0 & 2.58 & $\mathrm{ND}+\mathrm{NE}$ & 96.0 & 0.650 \\
\hline 22. & 43 & 15,000 & 15.0 & 10.0 & 3.72 & $\mathrm{ND}+\mathrm{NE}$ & 84.0 & 0.738 \\
\hline 23. & 55 & 4,000 & 14.0 & 1.0 & 3.90 & $N D+I C$ & 91.2 & 0.688 \\
\hline 24. & 52 & 30,000 & 11.0 & 8.0 & 3.10 & $N D+I C$ & 88.8 & 0.700 \\
\hline
\end{tabular}

ND: Newcastle disease, RW: Round worm, TW: Tape worm, NE: Necrotic enteritis, IC: Intestinal coccidiosis

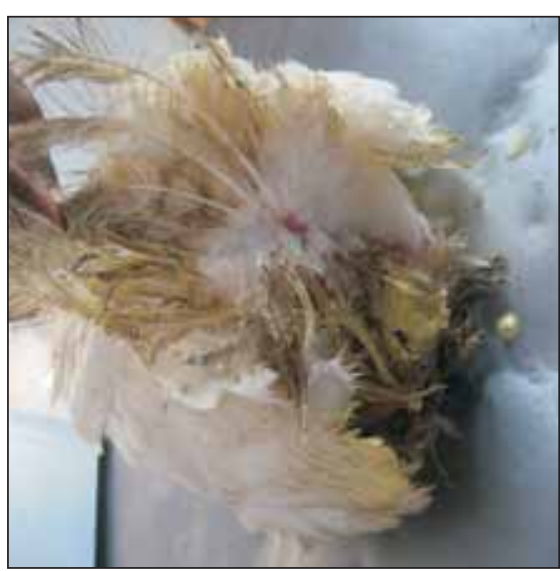

Figure-1: ND: Bird showing whitish to greenish diarrhoea

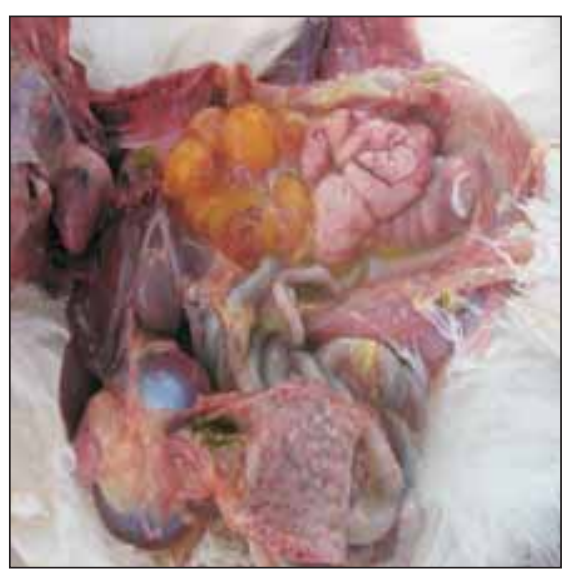

Figure-2: ND: Petechial haemorrhages in the proventriculus with haemorrhagic and degenerated ovarian follicles.

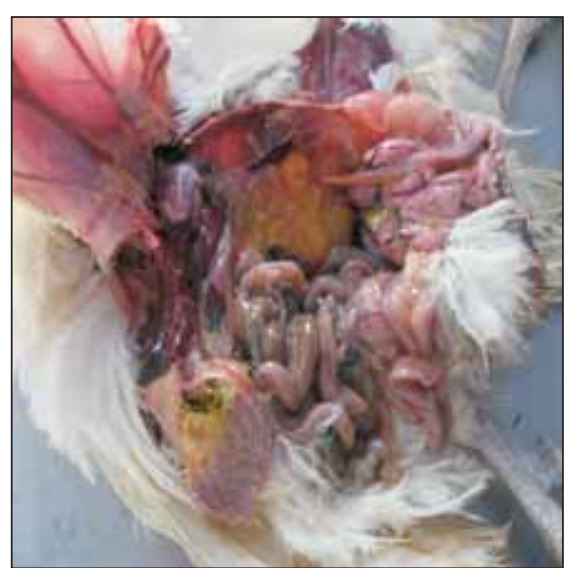

Figure-3: ND: Ovarian follicles were haemorrhagic, falccid and ruptured resulting in egg yolk peritonitis. materials were noticed. Carcasses were emaciated, dehydrated and pectoral muscles were congested. Petechial haemorrhages were observed on the mucosa of proventriculus around the papillary orifices and on caecal tonsils (Figure-2). Segmental to diffuse congestion were noticed on the intestinal mucosa especially in duodenum and ileum and the contents appeared as greenish and watery in nature. In majority of the cases marked degeneration of ovarian follicles were characterized by the flaccid and ill defined external outlines, congested and haemorrhagic follicles with egg yolk peritonitis (Figure-3). Oviduct serosal blood vessels were congested. Spleen and kidneys were congested, enlarged and stippled in appearance. In few birds mild to moderate congestion of lungs and trachea were noticed. Liver was slightly congested and friable in consistency. Gall bladder was distended with greenish yellow watery bile. In concurrent infections with worm infestation, necrotic enteritis and coccidiosis birds showed the lesions of ND along with the specific infections.
All the birds from investigated flocks were administered against Newcastle disease with $\mathrm{B}_{1}$ or $\mathrm{F}_{1}$ strain on $7^{\text {th }}$ day (intraocular route), Lasota strain on $5^{\text {th }}$ wk (intraocular route), Komarov or $\mathrm{R}_{2} \mathrm{~B}$ strain on $8^{\text {th }}$ wk (intramuscular or subcutaneous route)and Lasota (intraocular route) with ND Lasota killed vaccine (Subcutaneous route) at the point of lay. The HI titer of the investigated flocks were tested and found to be in the range of $1: 64$ to $1: 256$ on $3 \mathrm{wk}$ after vaccination. However the titer was increased to 1:512 to 1: 1024 from ailing birds at the time of disease outbreak. Newcastle disease virus was detected in 14 flocks $(58.33 \%)$ as a single infection and 10 flocks $(41.67 \%)$ as combined infections with worm infestation, necrotic enteritis and coccidiosis (Table-1). The incidence of ND was noticed in all age groups of layer chicken, however most commonly noticed in the early layers (21-50 wk). Most of the NDV outbreaks were recorded in the months of September and November (12 flocks; $50.0 \%$ ) followed by March and May (8 flocks; 33.33 
$\%$ ) and December and February (4 flocks; 16.67\%). Morbidity in the NDV and concurrently infected flocks varied from 5 to $10 \%$ and 5 to $15 \%$. Egg production drop from the expected level ranged between 3 to $7 \%$ in ND and 5 to $10 \%$ in concurrent infections. Mortality rate in NDV affected flocks ranged from 1.45 to $4.83 \%$ with an average mortality of $2.89 \%$. Average mortality observed in flocks with concurrent infection such as necrotic enteritis, round worm infestation, tape worm infestation and coccidiosis were 2.10, 2.68, 3.10 and $3.50 \%$ respectively.

\section{Discussion}

Newcastle disease is a global disease of economic importance and regarded as a very significant pathogen for domestic chickens. The virus is capable of infecting a large number of avian species with varying degrees of clinical manifestation in different age groups, however, relatively little is known about the clinical manifestation and prevalence of pathotypes in commercial layer chickens above 20 wk of age showing gastrointestinal lesions. In the present study, Newcastle disease was found to be one of the predominant diseases of gastrointestinal tract and it was noticed in $24 \%$ of the flocks with gastrointestinal lesions. Common occurrence of this disease among layers in Namakkal region has been supported by earlier workers $[8,9]$.

Allantoic fluid from embryos inoculated with pooled organ samples gave positive reaction in haemagglutination test. Haemagglutinating activity of these samples was inhibited by NDV specific hyperimmune serum in HI test, which is confirmatory for NDV [2]. In this study a better viral isolation was reached when samples were subjected to three passages in embryonated eggs. Contradictially, Kouwenhoven [10] observed that $85 \%$ of the positivity in the $1^{\text {st }}$ passage, only $10 \%$ needed a second blind passage and in exceptional cases, three blind passages were needed. This variation may be due to the nature and virus titer of sample used which plays an important role in the virus isolation.

On the basis of the pathogenicity of NDV in chickens, the viruses are classified into 3 main pathotypes: highly virulent (velogenic), intermediately virulent (mesogenic), and avirulent (lentogenic) [2, 6]. Analysis of $\mathrm{F}$ protein cleavage site of NDV is a favourable method to distinguish virulent and avirulent NDVs. However, since both velogenic and mesogenic NDVs share similar virulent type of $F$ cleavage site motifs, this method cannot be used to separate velogenic and mesogenic NDVs [11]. Thus, MDT and ICPI tests were carried out to distinguish the virulence of the isolated strains. Hanson and Brandly [12] reported that the velogenic, mesogenic and lentogenic strains of NDV killed the embryo in about $60 \mathrm{hrs}, 60$ to $90 \mathrm{hrs}$ and more than $100 \mathrm{hrs}$ respectively. Alexander and Senne [13] reported that the NDV strains demonstrating ICPI values from 1.5 to $2.0,1.0$ to 1.5 and 0.2 to 0.5 may be classified as velogenic, mesogenic and lentogenic strains respectively. However any ICPI value above 0.7 is classified in the mesogenic to virulent range and virulent isolates usually from 1.54 to 2.0 [2]. The results of the present study revealed $70.84,20.83$ and $8.33 \%$ of isolates falls in the mesogenic, velogenic and lentogenic pathotype respectively.

The clinical signs of a highly virulent NDV infection in chickens can be divided into 2 pathotypes; Virulent strains that cause diarrhea and frequent hemorrhagic intestinal lesions are called viscerotropic velogenic NDVs. On the other hand, strains that cause respiratory and neurotropic signs are called neurotropic velogenic NDVs [14]. In this study NDV affected birds showed mild to moderate symptoms related to gastrointestinal tract (anorexia and diarrohea) and drop in egg production, but not any signs specifically related to respiratory system [2]. Predominant postmortem lesions observed in the present study include matting of vent feathers, petechial haemorrhage on the tip of the proventricular papilla and caecal tonsils, congestion of duodenal and ileal mucosa and degeneration of ovarian follicles. The gross lesions observed in this study were suggestive of ND $[15,16]$ but not pathognomonic [2]. In this study, the clinical signs and lesions in the vaccinated chickens were mild and mainly restricted to gastrointestinal tract which indicates that the vaccination protects the birds from severe clinical signs and mortality and not infection and replication of the virus in the tissues [17]. This presents a bigger problem as it may mask the possible introduction and spread of virulent virus which become endemic but only become apparent when flock immunity level is down.

Among the 100 flocks showing gastrointestinal tract abnormalities 24 (24\%) found positive for NDV. This observation was concurs with Mukhopadhyay et al. [18] who also observed $29.71 \%$ of NDV infection in Namakkal area. The disease was recorded as single $(58.33 \%)$ and concurrent occurrence $(41.67 \%)$ with worm infestation, necrotic enteritis and coccidiosis. Newcastle disease virus causes lymphocyte necrosis, depletion in lymphoid organs $[19,20]$, and apoptosis of peripheral blood lymphocytes and mononuclear cells $[21,22]$, making the birds susceptible to concurrent infection.

Data obtained from this study showed that $50 \%$ of ND outbreaks in layers occurred among those between the point of lay and 6 months into production. According to FAO [23], the production cycle for most good breeds of layers found in both the temperate and tropical zones last for 17 months ( 72 weeks) under good management practices. Therefore, the $50 \%$ value of clinical ND obtained for the period of 6 months can be considered to be meaningful when compared with the total production cycle. In young birds initiation and peak egg production period produces considerable physiological and hormonal stress might be a contributing factor for their susceptibility to ND [24]. However the clinical manifestations and lesions were mild in young layers even if affected with velogenic strains except the 
drop in egg production due to high antibody titer due to vaccination. Commercial layer in this region are vaccinated against $\mathrm{ND}$ with $\mathrm{B}_{1}$ or $\mathrm{F}_{1}$ strain on $7^{\text {th }}$ day, Lasota strain on $5^{\text {th }}$ wk, Komarov or $\mathrm{R}_{2} \mathrm{~B}$ strain on $8^{\text {th }}$ wk and Lasota with ND Lasota killed vaccine at the point of lay with the aim to ensure the protection of laying hens until the end of peak laying period [5].

The results of the present study showed a high prevalence during September and November (50\%). Another relatively increased prevalence was noticed during March to May (33.33 \%). Stress associated with harsh weather conditions has been suggested to worsen the outcome of the ND [25]. During the above mentioned period Namakkal poultry zone experience frequent fluctuation in ambient temperature, humidity and other weather conditions which act as stress factors and lower the immune status of the birds making it possible for ND to manifest in commercial birds that have ordinary or lower herd immunity to the disease.

Egg production drop was noticed in all pathotypes and it ranged from 3 to $7 \%$ in ND and 5 to $10 \%$ in concurrent infection. This might be due the degeneration of follicles leads to arrest of ovulation and subsequent oviposition or temporary damage of the lining and glandular epithelium of the oviduct [9]. Average mortality recorded in NDV affected (2.89\%) and concurrently infected (3.50 in NDV and coccidiosis) flocks was lower than rates reported by Srithar et al. [26] who reported $17.58 \%$ mortality due to ND. The comparatively lower mortality recorded in this study could probably be attributed to the implementation of effective measures for the prevention of diseases by vaccination and maintenance of improved hygienic condition in cage system.

\section{Conclusion}

The present study revealed the prevalence of various pathotypes in vaccinated commercial layer chickens with gastrointestinal lesions and to control the disease strict biosecurity measures along with the vaccination is needed.

\section{Authors' contributions}

The present article is part of PB's Ph.D., research work. PB conceived and implemented the work. GAB was the major research supervisor. PS and SS were the minor research supervisors who made critical suggestions in carry out the study. All authors participated in draft of the manuscript. TRG critically reviewed the manuscript. All authors read and approved the final manuscript.

\section{Acknowledgements}

The authors are thankful to Tamil Nadu Veterinary and Animal Sciences University, Chennai, India for necessary fund and facilities provided for this study.

\section{Competing interests}

The authors declare that they have no competing interests.

\section{References}

1. Al-Garib, S. O., Gielkens, A. L. J. and Koch, G. (2003) Review of Newcastle disease virus with particular references to immunity and vaccination. World's Poul. Sci. J. 59: 185200.

2. Alexander, D. J. (2008) Newcastle disease, other avian paramyxoviruses, and pneumovirus infection. In: Disease of poultry, $12^{\text {th }}$ ed. (Shaif, Y.M., Barnes, H.J., Glisson, J.R. and McDougald, L.R.) Blackwell, Oxford, UK. p75-100.

3. Stram, Y., Shchori, D., Chinitch, Y., David, D., Molad, T. and Samina, I. (1998) Molecular characterization of an unassigned Israeli Newcastle disease virus isolate. Avian Dis. 42:746-51.

4. Bennejean, G. (1988) Newcastle disease: Control policies. In D.J. Alexander (ed). Newcastle Disease. Kluwer academic Publisher: Boston, MA, p303-317.

5. Srinivasan, P., Balasubramaniam, G.A., Gopalakrishnamurthy, T.R. and Balachandran, P. (2012) Pathology of oviduct in suboptimally producing commercial layer chicken. Int. J. Poult. Sci. 11:577-581.

6. World Organization for Animal Health (OIE). (2012) Chapter 2.3.14. Newcastle disease In: Manual of diagnostic tests and vaccines for terrestrial animals. World Organization for Animal Health (OIE), Paris. p1-19.

7. Pedersen, J. C. (2011) Procedure for Determining Mean Death Time for Newcastle Disease Virus Isolates, SOP-AV2016, National Veterinary Services Laboratories testing protocol, Ames, Iowa, USA.

8. Pazhanivel, N., Balasubramaniam, G.A., Titus George, V. and Mohan, B. (2002) Study of Natural outbreak of Newcastle Disease in and around Namakkal. Indian Vet. J., 79: 293 - 294.

9. Srinivasan, P. (2008) Pathology of Reproductive organs with special reference to oviduct in layer. Ph.D., thesis submitted to Tamil Nadu Veterinary and Animal Sciences University, Chennai.

10. Kouwenhoven, B. (1993) Newcastle disease. Pp.341-361. In: Virus Infections of Birds (McFerran, J.B. and McNulty, M.S.) Elsevier Science Publishers B.V, Amsterdam, Netherlands.

11. Adi, A.A.A.M., Astawa, N.M., Putra, K.S.A., Hayashi, Y. and Matsumoto, Y. (2010) Isolation and Characterization of a Pathogenic Newcastle Disease Virus from a Natural Case in Indonesia. J. Vet. Med. Sci. 72:313-319.

12. Hanson, R.P. and Brandly, C.A. (1955) Identification of vaccine strains of Newcastle disease virus. Science 122:156157.

13. Alexander, D.J. and Senne, D.A. (2008) Newcastle disease virus and other avian paramyxoviruses. In A laboratory manual for the isolation and identification of avian pathogens, $6^{\text {th }}$ ed (Dufour Zavala, L., Swayne, D.E., Glisson, J.R., Pearson, J.E., Reed, W.M., Jackwood, M.W. and Woolcock,P.R,). The American Association of Avian Pathologists, Athens, Georgia. p135-141.

14. Alexander, D.J. and Allan, W.H. (1974). Newcastle disease virus pathotypes. Avian Pathol. 3:269-278.

15. Parade, L. and Young, P.L. (1990) The pathogenesis of velogenic Newcastle disease virus infection in chickens of different ages and different levels of immunity. Avian Dis, 34: $803-808$.

16. Hamid, H., Campbell, R.S.F. and Parede, L. (1991) Studies on the Pathology of Velogenic New castle disease virus infection on non-immune and immune birds. Avian Pathol. 20: $561-575$.

17. Sumithra, A., Srinivasan, P., Balasubramaniam, G.A., Gopalakrishnamurthy, T.R. and Balachandran, P. (2013) Ameliorative effect of Panchagavya on Newcastle disease in layer chicken. Inter. J. Agri. Biosci., 2: 60-63.

18. Mukhopadhyay, H.K., Doriarajan, N., Titus George, V. and Chandran, N.D.J. (2002) Pathology of reproductive organs of layer birds in Newcastle disease infection. Indian J. Anim. Sci., 72:973-974. 
19. Masti, J., Nabni, C., Van den Berg, J. and Meulemans, G. (2005) Ultrastructural changes of the tracheal epithelium after vaccination of day old chicken with the Lasota strain of NDV. Vet. Pathol. 42: 559 - 565.

20. Agoha, N. J., Akpavie, S. O., Durojaiye, O. A. and Adene, D. F. (1992) Pathogenicity of two strains of Newcastle disease virus in the grey-breasted helmet guinea fowl. Vet. Quart. 14:51-53.

21. Lam, K. M. and Vasconcelos, A. C. (1994) Newcastle disease virus-induced apoptosis in chicken peripheral blood lymphocytes. Vet. Immunol. Immunopathol. 44:45-56.

22. Lam, K. M. (1996) Newcastle disease virus-induced apoptosis in the peripheral blood mononuclear cells of chickens. J. Comp. Pathol. 114:63-71.
23. Food and Agriculture Organisation (2003) Egg Production: Egg Production cycle. FAO Services Bulletin. FAO. Rome. p1-16.

24. Foilb, A.R., Lui, P. and Romeo, R.D. (2011) The transformation of hormonal stress responses throughout puberty and adolescence. J. Endocrinol. 210:391-398.

25. Abdu, P.A., Mera, U.M. and Saidu, L. (1992) A study of chicken mortality in Zaria, Nigeria. Proceeding of National Workshop on Livestock and Vet. Services, (NWLVS'92), National Veterinary Research Institute Vom, p51-55.

26. Srithar, A., Shoba, K., Saminathan, P., Chandran, N.D.G., Dorairajan, N. and Venugoplan, A.T. (1997) Pattern of mortality in commercial layer farms at Namakhal. Indian Vet.J., 74: 996-997.

$* * * * * * * *$ 